Pathologe 2008 · [Suppl 2] 29:184-186 DOI 10.1007/s00292-008-1035-x

Online publiziert: 29. August 2008

c) Springer Medizin Verlag 2008

\section{H. Moch}

Institut für Klinische Pathologie, Departement Pathologie,

UniversitätsSpital Zürich, Schweiz

\title{
Molekulare Basis der zielgerichteten Therapien bei metastasierenden Nierenzellkarzinomen
}

\section{Klinische Studien mit Sunitinib bzw. Sorafenib}

2007 publizierten Motzer et al. $[17,18]$ und Escudier et al. [2] Resultate von Phase-IIIStudien mit 2 oral applizierbaren, so genannten „Small-molecule-Kinaseinhibitoren“, Sunitinib bzw. Sorafenib. Beide Substanzen verbesserten das progressionsfreie Überleben von Patienten mit metastasierenden klarzelligen Nierenzellkarzinomen (kNZK). Weder Sunitinib noch Sorafenib hatten allerdings einen Einfluss auf das allgemeine Überleben. Obwohl beide Substanzen bedeutsame Nebenwirkungen hatten, wurden sie für die Behandlung des metastasierenden NZK zugelassen.

Das Ansprechen der klarzelligen Nierenzellkarzinome (kNZK) auf die Behandlung mit Sunitinib und Sorafenib wird auf die charakteristischen molekularen Veränderungen in diesen Tumoren zurückgeführt. In etwa $60-70 \%$ der kNZK, die in diesen Studien behandelt wurden, ist das von-Hippel-Lindau- (VHL-)Tumorsuppressorgen inaktiviert $[6,7,11,16,19,21]$. Das VHL-Protein (pVHL) reguliert verschiedene Transkriptionsfaktoren, insbesondere den Hypoxie-induzierbaren Faktor $\alpha(H I F-\alpha)$. Dieser wiederum ist wichtig für die Expression des Chemokinrezeptors CXCR4, der die Metastasierung der kNZK beeinflusst [22]. Die Regulation von HIF-a in den $V H L$-defizienten Tumorzellen spielt eine kritische Rolle in der Entstehung von Nierenkarzinomen [12].
Die Beobachtung, dass die Inaktivierung von VHL mit einer erhöhten HIF- $\alpha$ Aktivität und damit einer verstärkten Expression des „Vascular Endothelial Growth Factors A“ (VEGF), des „Platelet-derived Growth Factors b“ (PDGF b) und des „Transforming Growth Factor $\alpha$ “ (TGF- $\alpha$ ) auslöst, kann die Wirksamkeit der genannten Substanzen in kNZK erklären $[10,23]$. Prinzipiell ist die Blockade dieser Wachstumsfaktoren und angiogenetischer Faktoren wichtig, um das Überleben der Tumorzellen zu beeinflussen.

Ein Beispiel dafür ist die Wirkung von Bevacizumab, einem humanisierten monoklonalen Antikörper gegen VEGF. In einer kürzlich abgeschlossenen klinischen Studie mit Bevacizumab in Patienten mit metastasierenden NZK wurde in $10 \%$ der Patienten zumindest ein partielles Ansprechen beobachtet. Diese Behandlung führte damit zu einem signifikant verbesserten progressionsfreien Überleben [23]. Obwohl keine Verbesserung des allgemeinen Überlebens eintrat, zeigen diese Studien, dass die Blockade von VEGF das Tumorwachstum hemmen kann.

Die so genannten Small-moleculeKinaseinhibitoren Sunitinib und Sorafenib hemmen ebenfalls die von VEGF und PDGF b beeinflussten Proteine über die Blockade der Rezeptortyrosinkinasen VEGF-Rezeptor-2 und PDGF-Rezeptor- $\beta$ [3]. Die Beeinflussung dieser molekularen Signalwege in NZK bestätigt die Bedeutung von HIF in kNZK da HIF- $\alpha$ VEGF und PDGF $b$ reguliert. Eine ähn- liche Überlegung kann man für die Beeinflussung des TGF- $\alpha$-Signalweges anstellen, weil auch TGF- $\alpha$ überexprimiert wird, wenn VHL gehemmt ist. Dieser Signalweg führt zu einer Aktivierung von „Epidermal Growth Factor Receptor“ (EGFR), welcher in Nierenkarzinomen sehr stark exprimiert wird, obwohl EGFRAmplifikationen nicht oder allenfalls sehr selten auftreten $[14,15]$. Klinische Studien mit hemmenden Substanzen von TGF- $\alpha$ haben bisher keine signifikante Verbesserung des Überlebens zeigen können.

\section{Temsirolimus}

Kürzlich wurden die Ergebnisse einer Phase-III-Studie zum Kinaseinhibitor Temsirolimus publiziert [8]. Bei fortgeschrittenen und metastasierten NZK konnten Ansprechraten in etwa 9\% der Patienten beobachtet werden. Temsirolimus hemmt die „Mammalian Target of Rapamycin Complex 1 Kinase“ (mTOR; [9]). Über eine S6-Kinase-Hemmung kann mTOR die Translation von "Messenger-RNA“ (mRNA) aktivieren. Verschiedene Untersuchungen haben gezeigt, dass mTOR auch in der Entstehung von NZK bedeutsam ist. Primär reguliert mTOR auch HIF [9]. Zusätzlich wird die Aktivierung von mTOR über Mutationen der Gene des Tuberösen-Sklerose-Komplexes (TSC) 1 und 2 aktiviert [1]. Dies wiederum löst eine erhöhte HIF-Aktivität aus. In vitro konnte durch die Hemmung von mTOR die HIFAktivität nur reduziert werden, wenn vor- 
her mTOR aktiviert wurde [1]. Über den mTOR-Signalweg spielt möglicherweise auch das Tumorsuppressorgen Phosphatase und Tensin homolog (PTEN) eine Rolle. PTEN wird derzeit in verschiedenen Tumorprogressionsmodellen als wichtig für die Entstehung von Nierenkarzinomen eingestuft $[4,5]$.

\section{Ausblick}

Die neuen Therapieansätze erweitern heute die Therapieoptionen für Patienten mit metastasierten Nierenkarzinomen. Grundlage sind verschiedene Angriffspunkte in den durch die pVHL-Inaktivierung ausgelösten Signalkaskaden. Sunitinib und Sorafenib beeinflussen z. B. unterschiedliche Kinasen. Demgegenüber bindet Sirolimus spezifisch den mTORKomplex I. Sunitinib, Sorafenib und Temsirolimus beeinflussen gleichzeitig die Angiogenese und hemmen die Zellproliferationen der Tumorzellen. Die meisten der neuen Substanzen sind primär Inhibitoren der Angiogenese. Damit sind verschiedene Wirkmechanismen zu postulieren, die einerseits die Tumorzelle, andererseits aber auch die Gefäße der Tumoren betreffen ( $\bullet$ Abb. 1). Gerade die Angiogenese der Nierenkarzinome weist von Fall zu Fall große Unterschiede auf [13, 19], und Tumoren mit einer hohen Gefäßdichte haben eine eher günstige Prognose.

Ein wichtiges Ziel künftiger Untersuchungen ist die Identifikation von prädiktiven Markern für die Wirksamkeit der genannten Substanzen. Bei Lungenkarzinomen konnten molekulare Veränderungen identifiziert werden, die zwischen resistenten und nichtresistenten Tumoren unterscheiden [20]. Wenn es z. B. gelingen würde, spezifische VHL-Mutationen zu identifizieren, die das Ansprechen auf die genannten Medikamente vorhersagen helfen, könnte eine individualisierte Therapie für Nierenkarzinompatienten tatsächlich Wirklichkeit werden.

\section{Korrespondenzadresse \\ Prof. Dr. H. Moch}

Institut für Klinische Pathologie, Departement Pathologie, UniversitätsSpital Zürich

Schmelzbergstr. 12, 8091 Zürich

Schweiz

holger.moch@usz.ch

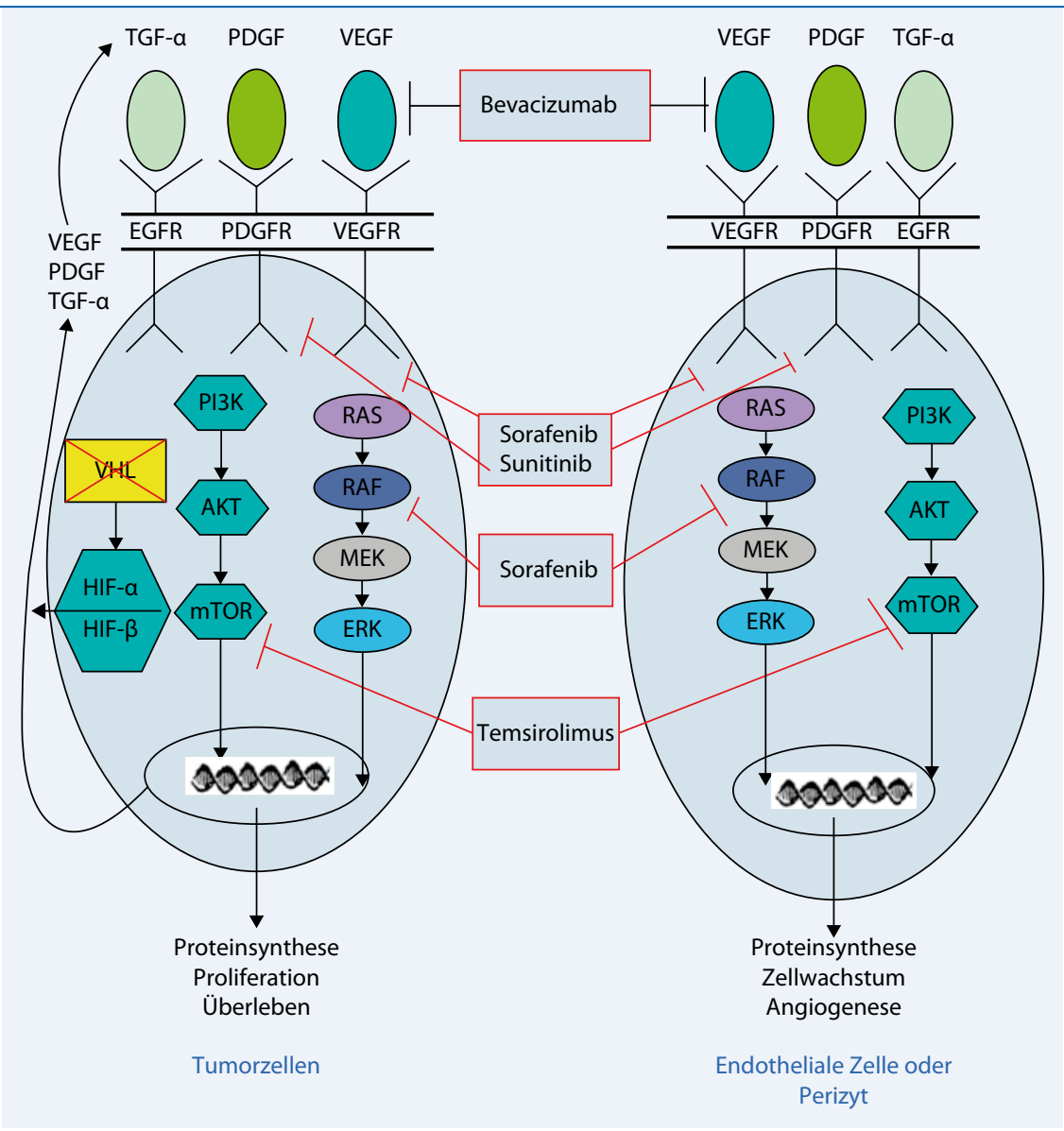

Abb. 1 \ Verschiedene Zielstrukturen von Tumorzellen und Gefäßen für die Behandlung des Nierenzellkarzinoms

Interessenkonflikt. Der korrespondierende Autor gibt an, dass kein Interessenkonflikt besteht.

\section{Literatur}

1. Brugarolas JB, Vazquez F, Reddy A et al. (2003) TSC2 regulates VEGF through mTOR-dependent and -independent pathways. Cancer Cell 4: 147158

2. Escudier B, Eisen T, Stadler WM et al. (2007) Sorafenib in advanced clear-cell renal-cell carcinoma. $\mathrm{N}$ Engl J Med 356: 125-134

3. Fabian MA, Biggs WH 3rd, Treiber DK et al. (2005) A small molecule-kinase interaction map for clinical kinase inhibitors. Nat Biotechnol 23: 329-336

4. Frew IJ, Minola A, Georgiev S et al. (2008) Combined Vhlh And Pten Mutation Causes Genital Tract Cystadenoma And Squamous Metaplasia. Mol Cell Biol 28: 4536-4548

5. Frew IJ, Thoma CR, Georgiev S et al. (2008) pVHL and PTEN tumour suppressor proteins cooperatively suppress kidney cyst formation. Embo J 27: 1747-1757

6. Gnarra JR, Tory K, Weng Y et al. (1994) Mutations of the VHL tumour suppressor gene in renal carcinoma. Nat Genet 7: 85-90

7. Herman JG, Latif F, Weng Y et al. (1994) Silencing of the VHL tumor-suppressor gene by DNA methylation in renal carcinoma. Proc Natl Acad Sci U S A 91: 9700-9704
8. Hudes G, Carducci M, Tomczak P et al. (2007) Temsirolimus, interferon alfa or both for advanced renal-cell carcinoma. N Engl J Med 356: 2271-2281

9. Hudson CC, Liu M, Chiang GG et al. (2002) Regulation of hypoxia-inducible factor 1alpha expression and function by the mammalian target of rapamycin. Mol Cell Biol 22: 7004-7014

10. Kaelin WG jr (2004) The von Hippel-Lindau tumor suppressor gene and kidney cancer. Clin Cancer Res 10: 6290S-6295S

11. Latif F, Tory K, Gnarra J et al. (1993) Identification of the von Hippel-Lindau disease tumor suppressor gene. Science 260(5112): 1317-1320

12. Maxwell PH, Wiesener MS, Chang GW et al. (1999) The tumour suppressor protein VHL targets hypoxia-inducible factors for oxygen-dependent proteolysis. Nature 399(6733): 271-275

13. Mertz KD, Demichelis F, Kim R et al. (2007) Automated immunofluorescence analysis defines microvessel area as a prognostic parameter in clear cell renal cell cancer. Hum Pathol 38: 14541462

14. Moch H, Sauter G, Buchholz N et al. (1997) Epidermal growth factor receptor expression is associated with rapid tumor cell proliferation in renal cell carcinoma. Hum Pathol 28: 1255-1259

15. Moch H, Sauter G, Gasser TC et al. (1998) EGF-r gene copy number changes in renal cell carcinoma detected by fluorescence in situ hybridization. $J$ Pathol 184: 424-429 
Pathologe 2008 · [Suppl 2] 29:184-186 DOI 10.1007/s00292-008-1035-x

(c) Springer Medizin Verlag 2008

\section{H. Moch \\ Molekulare Basis der zielgerichteten Therapien bei metastasierenden Nierenzellkarzinomen}

\section{Zusammenfassung}

In den letzten Jahren haben durch die Einführung der so genannten „targeted therapy" die patientenindividuellen Entscheidungsmöglichkeiten bei metastasierendem Nierenkarzinomen deutlich zugenommen. Grundlage dieser neuen Behandlungsmöglichkeiten ist die Inaktivierung des von-Hippel-Lindau- (VHL-)Tumorsuppressorgens. Dies resultiert in der Expression verschiedener proangiogener Wachstumsfaktoren, u. a. VEGF und PDGF. Basis der Therapie mit den neuen Medikamenten, z. B. Sorafenib, Sunitib und Temsirolimus, ist die Unterbrechung der Phosphorylierungskaskade in der Zelle, was zu einer Verhinderung der Signaltransduktion führt. Die Einsatzmöglichkeiten dieser Medikamente bei Patienten mit klarzelligen Nierenzellkarzinomen ( $k N Z K$ ) richten sich an bestimmten Signalwegen nach Inaktivierung von $V H L$ aus und müssen unter Berücksichtigung der Möglichkeiten, Grenzen und Nebenwirkungen der neuen Substanzen abgewogen werden. Ein prädiktiver Marker für das Ansprechen dieser „targeted therapy" existiert bislang allerdings noch nicht.

\section{Schlüsselwörter}

Nierenzellkarzinom · Kinaseinhibitoren . Metastasen $\cdot \mathrm{VHL} \cdot$ Targeted therapy

\section{Molecular basis of targeted therapy in metastatic renal cancer}

\section{Abstract}

The introduction of targeted therapy in metastatic renal cancer patients provides a whole array of individual therapeutic options. The basis for this treatment is the inactivation of the von Hippel-Lindau tumor suppressor gene, resulting in high expression of proangiogenic growth factors, e.g. vascular endothelial growth factor (VEGF) and plateletderived growth factor (PDGF). This provides the rationale for targeting these pathways in clear cell renal cell carcinomas by small molecule inhibitors. This article gives a review on clinical trials with sunitinib, sorafenib, and temsirolimus in patients with advanced renal cell carcinoma and shows how promising treatments can emerge from an understanding of the molecular genetics and signaling pathways of tumors. However, a predictive marker, e.g. specific mutations associated with drug-resistant or responsive tumors, has not yet been identified and is paramount for the future.

Keywords Renal cancer · Kinase inhibitors · Metastasis . $\mathrm{VHL} \cdot$ Targeted therapy
16. Moch H, Schraml P, Bubendorf L et al. (1998) Intratumoral heterogeneity of von Hippel-Lindau gene deletions in renal cell carcinoma detected by fluorescence in situ hybridization. Cancer Res 58: 2304-2309

17. Motzer RJ, Hutson TE, Tomczak P et al. (2007) Sunitinib versus interferon alfa in metastatic renal-cell carcinoma. N Engl J Med 356: 115-124

18. Motzer RJ, Rini Bl, Bukowski RM et al. (2006) Sunitinib in patients with metastatic renal cell carcinoma. JAMA 295: 2516-2524

19. Schraml P, Struckmann K, Hatz F et al. (2002) VHL mutations and their correlation with tumour cell proliferation, microvessel density and patient prognosis in clear cell renal cell carcinoma. J Pathol 196: 186-193

20. Sharma SV, Bell DW, Settleman J, Haber DA (2007) Epidermal growth factor receptor mutations in lung cancer. Nat Rev Cancer 7: 169-181

21. Shuin T, Kondo K, Torigoe S et al. (1994) Frequent somatic mutations and loss of heterozygosity of the von Hippel-Lindau tumor suppressor gene in primary human renal cell carcinomas. Cancer Res 54: $2852-2855$

22. Staller P, Sulitkova J, Lisztwan J et al. (2003) Chemokine receptor CXCR4 downregulated by von Hippel-Lindau tumour suppressor PVHL. Nature 425(6955): 307-311

23. Yang JC, Haworth L, Sherry RM et al. (2003) A randomized trial of bevacizumab, an anti-vascular endothelial growth factor antibody, for metastatic renal cancer. N Engl J Med 349: 427-434 